\title{
The rate of assimilation of visual information'
}

\section{ALAN ALLPORT, University of Aberdeen. Scotland}

The rate of uptake of information from a brief visual presentation was investigated by a method of backward masking (Sperling, 196.3) for two types of visual characters (digits and Landolt C's). The results showed that encoding of information from the Landolt C's occurred two to three times more slowly than for the overlearned, but more complex, numerals. Additional practice failed to reduce this disparity. A second experiment showed that it was not due to restricted cue size, although more severe size restriction did reduce still further the rate of uptake of information.

The information presented in a single brief exposure remains available to the $S$ in pre-perceptual storage for $0.25 \mathrm{sec}$ or more after the end of its physical exposure (Sperling, 1960; Averbach \& Sperling, 1961). Its availability can be effectively cut short, however, by the presentation during this interval of a second patterned stimulus whose contours fall on overlapping or closely adjacent positions on the retina (e.g., Kolers, 1962; Schiller, 1966), either because the second stimulus erases the first or simply sums with it to make the combined pattern unreadable. In either case, by varying the interval between onsets of the two stimuli, the time for which the first stimulus is available to the $S$ for processing can be experimentally controlled. Any information about it which is present in the S's report must then have been extracted from the visual display by the time the second, interfering stimulus occurs.

Particularly effective masking is provided by a visual "noise" field, consisting of densely scattered fragments of the test items. Using a row of letters as the test stimulus, immediately followed by such a visual "noise" field, Sperling (1963) found that the number of letters which could be reported correctly increased at the rate of about one letter per $10 \mathrm{msec}$ delay of the masking field for the first three or four letters, and thereafter at only about five letters per second. This initial very rapid increase in the accuracy of report is taken to indicate the rate at which such visual material can be coded into a symbolic (i.e., nonvisual) representation, ${ }^{2}$ such that it is no longer vulnerable to interference by the subsequent masking stimulus. ${ }^{3}$ The encoding operation appears to be broadly serial across discrete items, or positions, on the retina.

A question of considerable interest which arises out of these results and their possible interpretation is: Is the initial rate of uptake of information constant for different kinds of symbolic visual material, or does it vary with the type and difficulty of the coding processes involved? If it does so vary, certain relatively

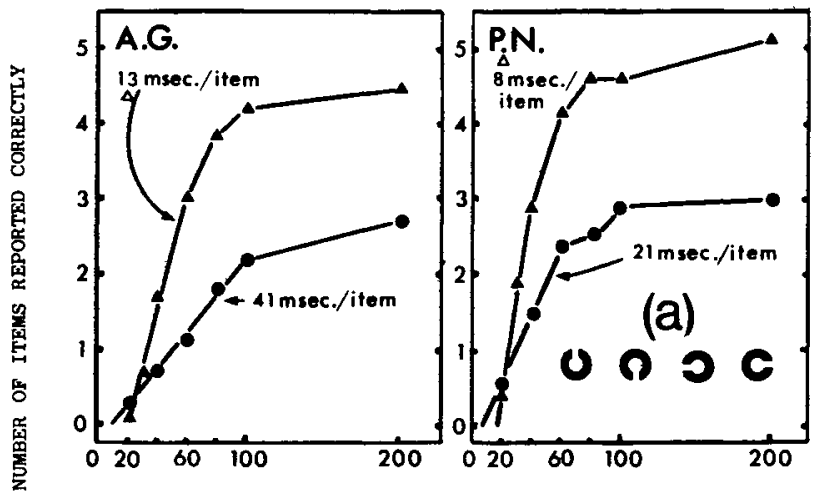

DELAY OF MASKING STIMULUS (MSEC.)

Fig. 1. Mean number of characters reported correctly as a function of delay of the post-exposure masking stimulus, i.e., time available for the intake of information, for two types of visual character: - - - Digits, - $-1-=$ Landolt Cs (see Fig. 1a). Representative results for two Ss. $\Delta=$ Digits, blank postexposure field. simple interpretations of this process may be rejected. The experiments described here provide a partial answer to this question.

\section{EXPERIMENT}

Method

Test items were (1) printed digits and (2) a vocabulary of four Landolt rings (Fig. I a). S's used the names "up," "down," "left," and "right" to indicate the position of the break in each ring. The test items were printed in black Letraset in a horizontal row 0.4 in. apart on white cards. The rings were 0.05 in. thick, outside diameter 0.20 in., break width 0.05 in. The digits were of similar dimensions (height 0.20 in., stroke width 0.04 in.). Stimuli were presented binocularly at a distance of $4 \mathrm{ft}$, in a Scientific Prototype GB 3-field tachistoscope. Luminance of pre-adapting, test, and masking fields (white area) was $18 \mathrm{ft}-\mathrm{L}$.

When ready, $S$ triggered the onset of the test exposure, which terminated with the onset of a masking field consisting of densely scattered fragments of the test items (digits or rings, respectively), covering an average $40 \%$ of the total field. A fresh stimulus card was inserted for each trial, and the test exposure varied between each block of 10 trials. $S$ spoke, and then wrote, his report after each trial. When uncertain he was encouraged to guess. Each $S$ had a preliminary 1-h practice session at both conditions. He then experienced the two conditions (digits or rings) on successive days, the order being counter-balanced between Ss. (Digits were also presented at $20 \mathrm{msec}$ exposure without masking, i.e., immediately followed by a return to the pre-adapting field.) Ss were six undergraduate and four graduate members of the psychology department, Aberdeen University, aged between 19 and 33 years (mean 23.6 years).

Results

Representative results for two Ss are shown in Fig. 1. Straight lines fitted by eye were used to estimate the initial rate of uptake of information for each S. Consistently for all Ss the initial rise in the accuracy of report occurred two to three times more slowly for the Landolt rings than far the digits: Mean $11.4 \mathrm{msec} / \mathrm{item}, \mathrm{SD}$ 3.0 , for digits vs $30.5 \mathrm{msec} / \mathrm{item}, \mathrm{SD} 11.7$, for the rings, $\mathrm{p}<.001$. Substantial differences in recognition rate were found between individuals, which were individually highly correlated over the two conditions $(r=0.91, p<.001)$. Changeover to a slower rate of gain of information occurred typically after 4-5 items for the digits vs only $2-3$ items for the rings, but not necessarily at the same exposure duration for both conditions. Without the aftercoming masking field $S s$ were able to read, on average, 4.5 digits at the 20 msec exposure (cf., Fig. 1).

\section{EXPERIMENT 2}

\section{Method}

This was carried out in order to check the possibility that the previous result was due simply to the physical dimensions of the cues concerned. Landolt rings were prepared with four different sizes of break: $0.0125,0.025,0.05$ (corresponding to the test items of Experiment 1), and 0.10 in. (Fig. 2a). Other details were as for the first experiment. Five paid undergraduate Ss experienced two practice sessions of $1 \mathrm{~h}$ each prior to the main experiment.

Results

Pooled results for all five Ss are shown in Fig. 2. Doubling the size of the break in the ring has little or no effect on the initial slope obtained (c. $30 \mathrm{msec} /$ item: open and filled circles). The difference between the recognition time required for these symbols and digits therefore cannot be due to restricted cue size. Halving the size of the break (open squares) and halving it again (diamonds) does however appear systematically to increase the processing time required per item.

\section{DISCUSSION}

There are a number of obvious differences between the two types of recognition item (digits or Landolt Cs), any or all of which could be responsible for the observed differences in the rate of uptake of information. (1) Landolt Cs vary in one feature 


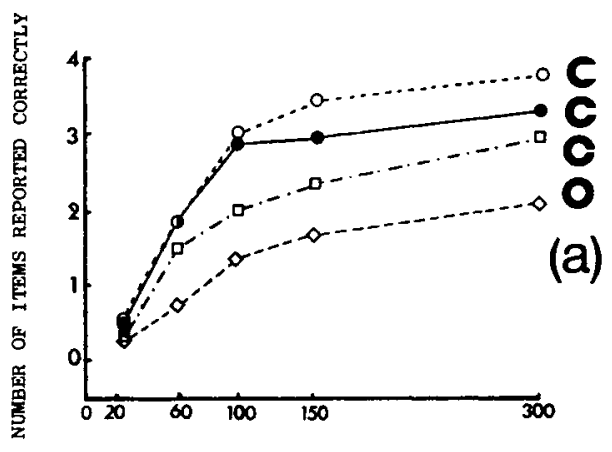

DELAY OF MASKING STIMULUS (MSEC.)

Fig. 2. Landolt C's: Effect of the size of break in the ring on the rate of intake of information. Mean of five S's. Solid circles show results for the same cue size as in Experiment 1.

only-the position of the break-whereas printed digits can be identified by multiple alternative cues. This would tend to give the advantage of speed to the digit vocabulary only if each item is scanned for a number of different features sequentially. If each item can be scanned for all relevant features (including all possible positions of the break) simultaneously, then it is difficult to see how cue-redundancy per se could cut down on the average time per item.

(2) Familarity: The naming-responses to the Landolt Cs appear to be less readily accessible than the highly overlearned digit names. Specific right-left confusions probably contribute to this difficulty. But while both tasks showed a slight increase over the first 100 trials in the number of items correct at any duration, this improvement was, if anything, slightly greater for the digits than for the Cs. The data of Experiment 2 were collected after Ss had already experienced over 2,000 test items, yet the mean recognition rate is not appreciably faster than in Experiment 1, where Ss received very much less practice. It seems unlikely, therefore, that achievable amounts of practice would eliminate the differences in recognition rate obtained between the two types of item. The results of Experiment 1 (digits), it may be noted, are closely similar to those reported by Sperling (1963) for capital letters.
Correct identification of 4.5 digits at only $20 \mathrm{msec}$ without backward masking demonstrates rather clearly the importance of thus restricting stimulus availability, where exposure duration is an experimental variable.

The present findings certainly raise more problems than they solve, but in a way which suggests that they may also be open to solution by extensions of the backward masking procedure. Further investigation, by this method, of recognition rates for stimuli varying for example in the dimensionality and redundancy of their critical features, as well as in the type of verbal label assigned to them, should provide some important clues towards the understanding of the visual recognition process.

\section{REFERENCES}

AVERBACH, E. \& SPERLING, G. Short term storage of information in vision. In C. Cherry (Ed.), Information theory. London: Butterworth, 1961, 196-211.

KOLERS, P. A. Intensity and contour effects in visual masking. Vision Research, 1962, 2, 277-294.

SCHILLER, P. H. Forward and backward masking as a function of relative overlap and intensity of test and masking stimuli. Perception \& Psychophysics, 1966, 1, 161-164.

SPERLING, G. The information available in brief visual presentations. Psychological Monographs, 1960, 74, No. 11 (Whole No. 498).

SPERLING, G. A model for visual memory tasks. Human Factors, 1963, 5, 19-31.

SPERLING, G. Successive approximations to a model for short-term memory. In A. F. Sanders (Ed.), Attention and performance, Amsterdam: North Holland Publishing Co. 1967.

\section{NOTES}

1. This research was supported in part by a grant from the Medical Research Council. The writer thanks Mrs. Alison Williamson for her assistance.

2. Sperling (1967) has suggested specifically as a "program of motor instructions" for verbal rehearsal.

3. The alternative suggestion, that the information has merely been transferred to a second stage of visual storage, without undergoing any other (non-trivial) coding transformation, seems highly uneconomical. Besides it is far from clear in that case why three or four letters should take longer to transfer than one letter. The changeover to a much slower rate of gain of information, as Sperling suggests, presumably defines a limit on immediate memory for such coded items. 